\title{
A COROLLARY OF THE B-FUNCTION LEMMA
}

\author{
A. BEILINSON AND D. GAITSGORY
}

\section{The STATEMENT}

1.1. Let $X$ be a smooth algebraic variety over an algebraically closed field $k$ of characteristic 0 . Let $f$ be a function on $X$; let $Y$ be the locus of zeros of $f$, and $j: U \hookrightarrow X$ the open embedding of the complement of $Y$. Let $\mathrm{D}_{X}$ be the sheaf of differential operators on $X$, and let $\mathcal{M}$ be a holonomic (left) D-module on $U$.

Let us tensor $\mathrm{D}_{X}$ with the ring of polynomials in one variable $k[s]$. I.e., let us consider the sheaf $\mathrm{D}_{X}[s]$, and the corresponding category of (left) $\mathrm{D}_{X}[s]$-modules (we follow the conventions in the theory of D-modules, where we only consider sheaves of $\mathrm{D}_{X^{-}}$or $\mathrm{D}_{X}[s]$-modules that are quasi-coherent as sheaves of $\mathcal{O}_{X}$-modules).

Consider now the $\mathrm{D}_{U}[s]$-module " $f s$ ". By definition, as $\mathcal{O}_{U}[s]$ module, it is free of rank one with the generator that we denote $f^{s}$, and vector fields acting on it by the formula

where $f^{s-1}:=f^{-1} \cdot f^{s}$.

$$
\xi\left(f^{s}\right)=s \cdot \xi(f) \cdot f^{s-1},
$$

Consider the $\mathrm{D}_{U}[s]$-module $\mathcal{M} \otimes " f s ":=\mathcal{M} \underset{\mathcal{O}_{U}}{\otimes} " f s "$, and the $\mathrm{D}_{X}[s]$-module

$$
j_{*}(\mathcal{M} \otimes " f s ") .
$$

It is easy to see that in general $j_{*}(\mathcal{M} \otimes " f s ")$ is not finitely generated as a $\mathrm{D}_{X}[s]-$ module:

Example. Consider $X=\mathbb{A}^{1}:=\operatorname{Spec}(k[t]), f=t, \mathcal{M}=\mathcal{O}_{X}$. Let $\tilde{\mathcal{M}}$ be the $\mathrm{D}_{X}[s]$ submodule of $j_{*}(" f s ")$, generated by the section $f^{s}$. It is easy to see that we have an isomorphism

$$
j_{*}(" f s ") / \widetilde{\mathcal{M}} \simeq \underset{n=0,1,2, \ldots}{\oplus}\left(\delta_{0} \otimes(k[s] / s-n)\right),
$$

where $\delta_{0}$ is the $\delta$-function at $0 \in \mathbb{A}^{1}$, thought of as a left D-module on $\mathbb{A}^{1}$, and $n \in \mathbb{N}$ is regarded as a point of $k \subset \operatorname{Spec}(k[s])$.

1.2. The goal of this note is to describe the set $\mathbf{V}(\mathcal{M})$ of all $\mathrm{D}_{X}[s]$-submodules $\widetilde{\mathcal{M}} \subset j_{*}(\mathcal{M} \otimes " f s ")$, such that $j^{*}(\widetilde{\mathcal{M}})=\mathcal{M} \otimes " f s "$, and the subset $\mathbf{V}_{f}(\mathcal{M}) \subset \mathbf{V}(\mathcal{M})$ that corresponds to those $\widetilde{\mathcal{M}}$ that are finitely generated as $\mathrm{D}_{X}[s]$-modules.

For $\widetilde{\mathcal{M}} \in \mathbf{V}(\mathcal{M})$ and a point $\lambda \in k \subset \operatorname{Spec}(k[s])$ consider the $\mathrm{D}_{X}$-module $\widetilde{\mathcal{M}}_{\lambda}:=$ $\widetilde{\mathcal{M}} /(s-\lambda)$. We have the canonical maps

$$
j_{!}\left(\mathcal{M} \otimes " f^{\lambda "}\right) \rightarrow \widetilde{\mathcal{M}}_{\lambda} \rightarrow j_{*}\left(\mathcal{M} \otimes " f^{\lambda "}\right),
$$

where $\mathcal{M} \otimes " f \lambda ":=\mathcal{M} \underset{\mathcal{O}_{U}}{\otimes} " f^{\lambda} "$ denotes the corresponding D-module over $U$.

Date: October 30, 2018. 
To state our main result, we shall adopt the following conventions. By an arithmetic progression in $k$ we shall mean a coset of $k$ modulo $\mathbb{Z}$. Let $\Lambda \subset k$ be a subset equal to union of finitely many arithmetic progressions. We say that some property of an element of $\Lambda$ holds for $\lambda \gg 0$ (resp., $\lambda \ll 0$ ), if it holds for elements of the form $\lambda_{0}+n$ for any fixed $\lambda_{0} \in \Lambda$, whenever $n \in \mathbb{Z}$ is sufficiently large (resp., small).

We now are ready to state our theorem:

Theorem 1. There exist a subset $\Lambda \subset k$ equal to the union of finitely many arithmetic progressions such that for any $\widetilde{\mathcal{M}} \in \mathbf{V}_{f}(\mathcal{M})$ we have:

(1) For $\lambda \notin \Lambda$ the maps

$$
j_{!}\left(\mathcal{M} \otimes " f^{\lambda "}\right) \rightarrow \widetilde{\mathcal{M}}_{\lambda} \rightarrow j_{*}\left(\mathcal{M} \otimes " f f^{\lambda "}\right)
$$

are isomorphisms. In particular, $\tilde{\mathcal{M}}_{\lambda} \simeq j_{! *}\left(\mathcal{M} \otimes " f^{\lambda "}\right)$.

(2) For $\lambda \in \Lambda$ with $\lambda \ll 0$, the map $\widetilde{\mathcal{M}}_{\lambda} \rightarrow j_{*}\left(\mathcal{M} \otimes ~ " f{ }^{\lambda "}\right)$ is an isomorphism.

(3) For $\lambda \in \Lambda$ with $\lambda \gg 0$, the map $j_{!}\left(\mathcal{M} \otimes ~ " f^{\lambda} "\right) \rightarrow \widetilde{\mathcal{M}}_{\lambda}$ is an isomorphism.

Note that assertion of the theorem provides an algorithm for computing $j_{!}(\mathcal{N})$. Namely, we must pick any finitely generated submodule $\tilde{\mathcal{M}} \subset j_{*}(\mathcal{M} \otimes ~ " f s ")$, such that $j^{*}(\tilde{\mathcal{M}}) \simeq \mathcal{M} \otimes " f s "$, and

$$
j_{!}(\mathcal{M}) \simeq \tilde{\mathcal{M}} / s-n
$$

for a sufficiently large integer $n$.

\section{A REFORMULATiON}

2.1. We shall derive Theorem 1 from a slightly more precise assertion. Before stating it, let us recall the following result, which is a well-known consequence of the b-function lemma (the proof will be recalled for completeness in the next section).

In what follows, if $P$ is a module over $k[s]$ and $\lambda$ is an element of $k \subset \operatorname{Spec}(k[s])$, we shall denote by $P_{(\lambda)}$ the localization of $P$ at the corresponding maximal ideal, i.e., $s-\lambda$.

We are going to study $\mathrm{D}_{X}[s]_{(\lambda)}$-submodules $\widetilde{\mathcal{M}}_{(\lambda)} \subset j_{*}(\mathcal{M} \otimes " f s ")_{(\lambda)}$ such that $j^{*}\left(\widetilde{\mathcal{M}}_{(\lambda)}\right)=\left(\mathcal{M} \otimes " f^{s "}\right)_{(\lambda)}$. We shall denote this set by $\mathbf{V}\left(\mathcal{M}{ }_{(\lambda)}\right)$.

Theorem 2. For any $\lambda \in k$ the following holds:

(A) The $\mathrm{D}_{X}[s]_{(\lambda)}$-module $j_{*}(\mathcal{M} \otimes " f s ")_{(\lambda)}$ is finitely generated. Denote it $\tilde{\mathcal{M}}_{(\lambda)}^{\max }$.

(B) The set $\mathbf{V}\left(\mathcal{M}_{(\lambda)}\right)$ contains the minimal element. Denote it $\tilde{\mathcal{M}}_{(\lambda)}^{\text {min }}$. Moreover, we have:

(B.1) The quotient $\tilde{\mathcal{M}}_{(\lambda)}^{\max } / \widetilde{\mathcal{M}}_{(\lambda)}^{\min }$ is $(s-\lambda)$-torsion.

(B.2) The natural map $j_{!}\left(\mathcal{M} \otimes " f^{\lambda "}\right) \rightarrow\left(\tilde{\mathcal{M}}_{(\lambda)}^{\text {min }}\right) / s-\lambda$ is an isomorphism.

(C) There exists a subset $\Lambda \subset k$ equal to the union of finitely many arithmetic progressions such for $\lambda \notin \Lambda, \widetilde{\mathcal{M}}_{(\lambda)}^{\min }=\tilde{\mathcal{M}}_{(\lambda)}^{\max }$. 
2.2. The strengthening of Theorem 1 mentioned above reads as follows:

Theorem 3. Let $\Lambda$ be as above, and let $\tilde{\mathcal{M}}$ be an element of $\mathbf{V}(\mathcal{M})$.

(I) For $\lambda \notin \Lambda$, the maps

$$
\tilde{\mathcal{M}}_{(\lambda)}^{\min } \rightarrow \tilde{\mathcal{M}}_{(\lambda)} \rightarrow \tilde{\mathcal{M}}_{(\lambda)}^{\max }
$$

are isomorphisms.

(II) The map $\tilde{\mathcal{M}}_{(\lambda)} \rightarrow \widetilde{\mathcal{M}}_{(\lambda)}^{\max }$ is an isomorphism for all $\lambda \in \Lambda$ that are $\ll 0$.

(III) The element $\tilde{\mathcal{M}}$ belongs to $\mathbf{V}_{f}(\mathcal{M})$ if and only if the map $\tilde{\mathcal{M}}_{(\lambda)}^{\text {min }} \rightarrow \tilde{\mathcal{M}}_{(\lambda)}$ is an isomorphism for all $\lambda \in \Lambda$ that are $\gg 0$.

2.3. Let us first see some obvious implications. First, point (C) of Theorem 2 implies point (I) of Theorem 3. Combined with point (B.2) of Theorem 2, point (I) of Theorem 3 implies point (1) of Theorem 1 .

Point (II) of Theorem 3 implies point (2) of Theorem 1, Point (III) of Theorem 3 , combined with point (B.2) of Theorem 2 implies point (3) of Theorem 1.

Finally, the "only if" direction Theorem 3 (III), combined with point (A) of Theorem 2] implies the "if" direction.

Furthermore, we have the following corollaries:

Corollary 1. Specifying an element $\widetilde{\mathcal{M}} \in \mathbf{V}(\mathcal{M})$ is equivalent to specifying, for each $\lambda \in \Lambda$, of an element $\tilde{\mathcal{M}}_{(\lambda)} \in \mathbf{V}\left(\mathcal{M}_{(\lambda)}\right)$, such that $\tilde{\mathcal{M}}_{(\lambda)}=\tilde{\mathcal{M}}_{(\lambda)}^{\text {max }}$ for all $\lambda$ that are $\ll 0$.

Corollary 2. Let $\tilde{\mathcal{M}}^{1}$ and $\tilde{\mathcal{M}}^{2}$ be elements of $\mathbf{V}_{f}(\mathcal{M})$. Then the localizations $\tilde{\mathcal{M}}_{(\lambda)}^{1}$ and $\widetilde{\mathcal{M}}_{(\lambda)}^{2}$ coincide for all but finitely many elements $\lambda \in k$.

2.4. We shall now give a description of the set $\mathbf{V}\left(\mathcal{M}_{(\lambda)}\right)$, appearing in Corollary 1 in terms of a vanishing cycles datum. With no restriction of generality, we can assume that $\lambda=0$.

Recall that Sect. 4.2 of [2] identifies the quotient $\tilde{\mathcal{M}}_{(0)}^{\max } / \tilde{\mathcal{M}}_{(0)}^{\min }$, which is a $\mathrm{D}_{X}[s]_{(0)}$-module set-theoritically supported on $Y=X-U$, with the D-module $\Psi^{\text {nilp }}(\mathcal{M})$ of nilpotent nearby cycles of $\mathcal{M}$, with the action of $s$ on it being the nilpotent "logarithm of monodromy" operator.

Thus, elements $\mathcal{N}$ of $\mathbf{V}\left(\mathcal{M}_{(0)}\right)$ are in bijection with $s$-stable $\mathrm{D}_{X}$-submodules

$$
\mathcal{K} \subset \Psi^{\text {nilp }}(\mathcal{M}) .
$$

For each $\mathcal{K}$ as above, let us describe more explicitly the corresponding $\mathrm{D}_{X^{-}}$ module $\mathcal{N}_{0}:=\mathcal{N} / s$. By [1], $\mathcal{N}_{0}$ is completely determined by the corresponding D-module of vanishing cycles $\Phi^{\text {nilp }}\left(\mathcal{N}_{0}\right)$, together with maps

$$
\Psi^{n i l p}(\mathcal{M}) \stackrel{\mathbf{c}}{\rightarrow} \Phi^{\text {nilp }}\left(\mathcal{N}_{0}\right) \stackrel{\mathbf{v}}{\rightarrow} \Psi^{\text {nilp }}(\mathcal{M})
$$

such that the composition $\mathbf{v} \circ \mathbf{c}: \Psi^{\text {nilp }}(\mathcal{M}) \rightarrow \Psi^{\text {nilp }}(\mathcal{M})$ equals $s$.

It is easy to see that $\Phi^{\text {nilp }}\left(\mathcal{N}_{0}\right)$ is given in terms of $\mathcal{K}$ by either of the following two expressions:

$$
\operatorname{coker}\left(\mathcal{K} \stackrel{\iota \oplus s}{\longrightarrow} \Psi^{n i l p}(\mathcal{M}) \oplus \mathcal{K}\right)
$$


or

$$
\operatorname{ker}\left(\Psi^{n i l p}(\mathcal{M}) / \mathcal{K} \oplus \Psi^{n i l p}(\mathcal{N}) \stackrel{s \oplus \pi}{\longrightarrow} \Psi^{n i l p}(\mathcal{M}) / \mathcal{K}\right),
$$

where $\iota: \mathcal{K} \hookrightarrow \Psi^{\text {nilp }}(\mathcal{N})$ and $\pi: \Psi^{\text {nilp }}(\mathcal{M}) \rightarrow \Psi^{\text {nilp }}(\mathcal{M}) / \mathcal{K}$ are the natural embedding and projection, respectively. The above kernel and co-kernel are identified by means of the map $\Psi^{n i l p}(\mathcal{M}) \oplus \mathcal{K} \rightarrow \Psi^{n i l p}(\mathcal{M}) / \mathcal{K} \oplus \Psi^{\text {nilp }}$ which has the following non-zero components:

$$
-s: \Psi^{n i l p}(\mathcal{M}) \rightarrow \Psi^{n i l p}(\mathcal{M}) ; \iota: \mathcal{K} \rightarrow \Psi^{n i l p}(\mathcal{M}) ; \pi: \Psi^{n i l p}(\mathcal{M}) \rightarrow \Psi^{n i l p}(\mathcal{M}) / \mathcal{K}
$$

The map $\mathbf{c}$ is the composition

$$
\Psi^{n i l p}(\mathcal{N}) \rightarrow \Psi^{n i l p}(\mathcal{N}) \oplus \mathcal{K} \rightarrow \Phi^{n i l p}\left(\mathcal{N}_{0}\right),
$$

and the map $\mathbf{v}$ is the composition

$$
\Phi^{n i l p}\left(\mathcal{N}_{0}\right) \rightarrow \Psi^{n i l p}(\mathcal{M}) / \mathcal{K} \oplus \Psi^{n i l p}(\mathcal{M}) \rightarrow \Psi^{n i l p}(\mathcal{M}) .
$$

We note that the !-restriction of $\mathcal{N}_{0}$ to $Y$ is then

$$
\operatorname{Cone}\left(\Psi^{n i l p}(\mathcal{M}) / \mathcal{K} \stackrel{s}{\rightarrow} \Psi^{n i l p}(\mathcal{M}) / \mathcal{K}\right)[-1],
$$

and the ${ }^{*}$-restriction of $\mathcal{N}_{0}$ to $Y$ is $\operatorname{Cone}(\mathcal{K} \stackrel{s}{\rightarrow} \mathcal{K})$.

\section{Proofs}

3.1. As all statements are local, we can assume that $X$ is affine. First, let us recall the statement of the usual b-function lemma:

Lemma 1. (J. Bernstein) Let $\mathcal{N}$ be as in Sect. 1.1, and let $m_{1}, \ldots, m_{n}$ be generators of $\mathcal{M}$ as a $\mathrm{D}_{U}$-module. Then there exist elements $P_{i, j} \in \mathrm{D}_{X}[s]$ and an element $\mathbf{b} \in k[s]$ such that for every $i$

$$
\Sigma_{j} P_{i, j}\left(m_{j} \otimes f^{s}\right)=\mathbf{b} \cdot\left(m_{i} \otimes f^{s-1}\right) .
$$

Let us deduce some of the statements of Theorems 2 and 3

3.2. First, it is clear that for $\lambda \in k$ and $n \in \mathbb{Z}$ such that

$$
((\lambda-n)-\mathbb{N}) \cap \operatorname{roots}(\mathbf{b})=\emptyset,
$$

the elements $m_{i} \otimes f^{s-n}$ generate $j_{*}(\mathcal{M} \otimes " f s ")_{(\lambda)}$ as a $\mathrm{D}_{X}[s]_{(\lambda)}$-module. This implies point (A) of Theorem 2 .

Set

$$
\Lambda=\mathbb{Z}+\operatorname{roots}(\mathbf{b}) .
$$

Point (C) of Theorem 2 and point (II) of Theorem 3 follow as well.

3.3. Note that we also obtain that the $\mathrm{D}_{X} \otimes k(s)$-module $j_{*}(\mathcal{M} \otimes ~ \otimes f s ") \underset{k[s]}{\otimes} k(s)$ does not have proper submodules, whose restriction to $U$ is $(\mathcal{M} \otimes ~ " f s ") \underset{k[s]}{\otimes} k(s)$.

This proves point (B.1) of Theorem 2 modulo the existence of $\widetilde{\mathcal{M}}_{(\lambda)}^{\text {min }}$. 
3.4. To prove point (B) of Theorem 2 and the remaining "only if" direction of Theorem 3(III), we shall use a duality argument.

Let $A$ be a localization of a smooth $k$-algebra (we shall take $A$ to be either $k[s]$ or $k[s]_{(\lambda)}$, or $\left.k(s)\right)$. Let $n=\operatorname{dim}(X)$. Consider the ring $\mathrm{D}_{X} \otimes A$.

Let $D_{c o h}^{b}\left(\mathrm{D}_{X} \otimes A\right.$-mod) (resp., $\left.D_{c o h}^{b}\left(\bmod -\mathrm{D}_{X} \otimes A\right)\right)$ denote the bounded derived category of left (resp., right) $\mathrm{D}_{X} \otimes A$-modules with coherent cohomologies.

Consider the contravariant functor

$$
\mathbb{D}_{A}: D_{c o h}^{b}\left(\mathrm{D}_{X} \otimes A \text {-mod }\right) \rightarrow D_{c o h}^{b}\left(\mathrm{D}_{X} \otimes A \text {-mod }\right),
$$

defined by composing the contravariant functor

$$
\mathcal{M} \mapsto \operatorname{RHom}\left(\mathcal{M}, \mathrm{D}_{X} \otimes A\right),
$$

which maps

$$
D_{\text {coh }}^{b}\left(\mathrm{D}_{X} \otimes A \text {-mod }\right) \rightarrow D_{\text {coh }}^{b}\left(\bmod -\mathrm{D}_{X} \otimes A\right),
$$

followed by tensor product with $\omega_{X}^{-1}[n]$ that maps $D_{c o h}^{b}\left(\bmod -\mathrm{D}_{X} \otimes A\right)$ back to $D_{c o h}^{b}\left(\mathrm{D}_{X} \otimes A\right.$-mod). The same argument as in the case of usual D-modules shows that $\mathbb{D}_{A} \circ \mathbb{D}_{A} \simeq \mathrm{Id}$.

We have the following basic property of the functor $\mathbb{D}_{A}$ : let $A \rightarrow B$ be a homomorphism between $k$-algebras, and let $\mathcal{N}$ be an object of $D_{\text {coh }}^{b}\left(\mathrm{D}_{X} \otimes A\right.$-mod). We have:

$$
\mathbb{D}_{B}(B \stackrel{L}{\otimes} \mathcal{N}) \simeq B \underset{A}{\stackrel{L}{\otimes}} \mathbb{D}_{A}(\mathcal{N})
$$

In particular, for $\mathcal{M} \in D_{\text {coh }}^{b}\left(\mathrm{D}_{X}\right.$-mod $)$, we have $\mathbb{D}_{A}(\mathcal{M} \otimes A) \simeq \mathbb{D}(\mathcal{M}) \otimes A$, where $\mathbb{D}$ denotes the usual duality on $D_{\text {coh }}^{b}\left(\mathrm{D}_{X}\right.$-mod).

3.5. First, let us note that $\mathbb{D}_{k[s]}(\mathcal{M} \otimes ~ " f s ")$ is acyclic off cohomological degree 0 , and

$$
\mathbb{D}_{k[s]}(\mathcal{M} \otimes " f s ") \stackrel{\sigma}{\simeq} \mathbb{D}(\mathcal{M}) \otimes " f s ",
$$

where $\sigma$ means that the action of $k[s]$ on the two sides differs by the automorphism $\sigma: k[s] \rightarrow k[s], \sigma(s)=-s$.

Let now $\mathcal{N}$ be an element of $\mathbf{V}\left(\mathcal{M}_{(\lambda)}\right)$; in particular, $\mathcal{N}$ is finitely generated over $\mathrm{D}_{X}[s]_{(\lambda)}$ by Theorem 2(A). We shall prove:

\section{Lemma 2.}

(a) The $\mathrm{D}_{X}[s]_{(\lambda)}$-module $\mathbb{D}_{k[s]_{(\lambda)}}(\mathcal{N})$ is concentrated in cohomological degree zero.

(b) The canonical map

$$
\mathbb{D}_{k[s]_{(\lambda)}}(\mathcal{N}) \rightarrow j_{*}\left(\mathbb{D}_{k[s]_{(\lambda)}}\left((\mathcal{M} \otimes " f s ")_{(\lambda)}\right)\right) \stackrel{\sigma}{\simeq} j_{*}(\mathbb{D}(\mathcal{M}) \otimes " f s ")_{(-\lambda)}
$$

is an injection.

For the proof of the lemma see Sect. 3.7 below. 
3.6. End of proofs of the theorems. The above lemma implies point (B) of Theorem 2 and the "if" direction in Theorem 3(III):

For point (B) of Theorem 2, the sought-for submodule $\widetilde{\mathcal{M}}_{(\lambda)}^{\text {min }}$ is given by

$$
\mathbb{D}_{k[s]_{(\lambda)}}\left(j_{*}(\mathbb{D}(\mathcal{M}) \otimes " f s ")_{(-\lambda)}\right) .
$$

Point (B.2) follows from equation (11).

For a finitely generated submodule $\widetilde{\mathcal{M}}$ as in point (III) of Theorem 3 , the map

$$
\tilde{\mathcal{M}}_{(\lambda)}^{\min } \rightarrow \tilde{\mathcal{M}}_{(\lambda)}
$$

is an isomorphism whenever the corresponding map

$$
\left(\mathbb{D}_{k[s]}(\tilde{\mathcal{M}})\right)_{(-\lambda)} \rightarrow j_{*}(\mathbb{D}(\mathcal{M}) \otimes " f s ")_{(-\lambda)}
$$

is an isomorphism.

3.7. Proof of Lemma 2, We shall use the following corollary of Lemma 1, established in [3]:

Corollary 3. The $\mathrm{D}_{X} \otimes k(s)$-module $j_{*}(\mathcal{M} \otimes ~ " f s ") \underset{k[s]}{\otimes} k(s)$ is holonomic.

From the corollary, we obtain that non-zero cohomologies of $\mathbb{D}_{k[s]_{(\lambda)}}(\mathcal{N})$ are $s$ torsion. Hence, to prove point (a), it is enough to show that

$$
k \underset{k[s]_{(\lambda)}}{\otimes} \mathbb{D}_{k[s]_{(\lambda)}}(\mathcal{N})
$$

is acyclic off cohomological degree 0 .

This acyclicity would also imply that $\mathbb{D}_{k[s]_{(\lambda)}}(\mathcal{N})$ has no $s$-torsion. Combined with Sect. 3.3 this would imply point (b) of the lemma as well.

Using isomorphism (11), the acyclicity of (2) is equivalent to $k \underset{k[s]_{(\lambda)}}{\otimes} \mathcal{L}=: \mathcal{N}_{\lambda}$ being holonomic. The latter is true for $\mathcal{N}=j_{*}(\mathcal{M} \otimes " f s ")_{(\lambda)}$, since in this case $\mathcal{N}_{\lambda} \simeq j_{*}\left(\mathcal{M} \otimes " f^{\lambda "}\right)$, which is known to be holonomic.

For any $\mathcal{N}$ we argue as follows. We note that $j_{*}(\mathcal{M} \otimes " f s ")_{(\lambda)} / \mathcal{N}$, being finitely generated over $\mathrm{D}_{X} \otimes k[s]_{(\lambda)}$ and $(s-\lambda)$-torsion, is finitely generated over $\mathrm{D}_{X}$. Since $\left(j_{*}(\mathcal{M} \otimes " f s ")_{(\lambda)} / \mathcal{N}\right) / s-\lambda$ is holonomic, being a quotient of $j_{*}(\mathcal{M} \otimes " f s ")_{(\lambda)} / s-\lambda$, we obtain that $j_{*}(\mathcal{M} \otimes " f s ")_{(\lambda)} / \mathcal{N}$ is itself holonomic as a $\mathrm{D}_{X}$-module.

We have a map

$$
\mathcal{N}_{\lambda} \rightarrow j_{*}\left(\mathcal{M} \otimes " f^{\lambda "}\right),
$$

whose kernel and cokernel are subquotients of $j_{*}(\mathcal{M} \otimes " f s ")_{(\lambda)} / \mathcal{N}$, which implies that $\mathcal{N}_{\lambda}$ is holonomic as well. 
3.8. An alternative argument. We can prove that $\mathbb{D}_{k[s]_{(\lambda)}}(\mathcal{N})$ lies in cohomological degree 0 directly, without quoting Corollary 3 . Namely, we have the following general assertion that follows from the usual Nakayama lemma:

Lemma 3. Let $B$ be a filtered $k$-algebra such that $\operatorname{gr}(B)$ is a commutative finitely generated algebra over $k$. Let $R$ be a localization of a commutative finitely generated $k$-algebra at a maximal ideal $\mathfrak{m}$. Then if $\mathcal{P}$ is a finitely generated $R \otimes B-$ module, such that $\mathcal{P} / \mathfrak{m} \cdot \mathcal{P}=0$, then $\mathcal{P}=0$.

Hence, Lemma 3 implies that the acyclicity of (2) implies that $\mathbb{D}_{k[s]_{(\lambda)}}(\mathcal{N})$ lies in cohomological degree 0, i.e., point (a) of Lemma 2

In particular, we can apply Lemma 2(a) to $j_{*}(\mathcal{M} \otimes " f s ")$, and isomorphism (11) to the homomorphism $k[s] \rightarrow k(s)$. We conclude that $\mathbb{D}_{k(s)}\left(j_{*}(\mathcal{M} \otimes " f s ") \underset{k[s]}{\otimes} k(s)\right)$ lies in cohomological degree 0 , i.e., that $j_{*}(\mathcal{M} \otimes " f s ") \underset{k[s]}{\otimes} k(s)$ is holonomic. This reproves Corollary 3 ,

\section{REFERENCES}

[1] A. Beilinson, How to glue perverse sheaves, in: $K$-theory, arithmetic and geometry (Moscow, 1984-1986), pp. 42-51, Lecture Notes in Math., 1289, Springer, Berlin, 1987.

[2] A. Beilinson, J. Bernstein, A proof of Jantzen's conjectures, in: I. M. Gel'fand Seminar, pp. 1-50, Adv. Soviet Math., 16, Part 1, Amer. Math. Soc., Providence, RI, 1993.

[3] J. Bernstein, Algebraic theory of D-modules, lecture notes, available as .ps file at http://www.math.uchicago.edu/ mitya/langlands.html

A.B.: Dept. of Math, The Univ. of Chicago, 5734 University Ave., Chicago IL, 60637

D.G.: Dept. of Math., Harvard Univ., 1 Oxford str., Cambridge MA, 02138

E-mail address: A.B.:sasha@math.uchicago.edu, D.G.:gaitsgde@math.harvard.edu 